\title{
Magnetic flux tubes observed with THEMIS/MSDP
}

\author{
P. Mein ${ }^{1}$, N. Mein ${ }^{1}$, M. Faurobert ${ }^{2}$, G. Aulanier ${ }^{1}$, and J.-M. Malherbe ${ }^{1}$ \\ 1 Observatoire de Paris, Section de Meudon, LESIA, 92195 Meudon Cedex, France \\ e-mail: Pierre.Mein@obspm.fr \\ 2 Université de Nice, UMR 6525, Parc Valrose, 06108 Nice Cedex, France
}

Received 23 May 2006 / Accepted 12 October 2006

ABSTRACT

\begin{abstract}
Aims. We use spectro-polarimetric THEMIS/MSDP data to investigate the 3D structure of solar magnetic-flux tubes across the upper photosphere.

Methods. Profiles of the sodium $\mathrm{D}_{1}$ line $589.6 \mathrm{~nm}$ are analysed by the bisector method at different wavelengths from the core to the wings, for several bright features. They are compared to synthetic profiles derived from 2D magnetic models of flux tubes and from the MULTI code for NLTE line profiles. Three different magnetic models of flux tubes are investigated. Model (I) consists of a single flux tube that compensates for the horizontal Lorentz forces exactly, while model (II) uses a compromise between horizontal and vertical components. Model (III), a conglomerate of thinner flux tubes, leads to the best agreement with observations.

Results. (1) The combination of seeing effects (small filling factor) with slopes of line profiles, which are different in the flux tubes and the neighbouring quiet sun, account for the decrease in observed magnetic field from line core to line wings in central parts of magnetic features, as well as the decrease in magnetic fluxes integrated over the whole magnetic features. (2) The expansion with height of single magnetic flux tubes (models I and II) accounts for the increase in the size of magnetic features from line wings to line core. (3) Pure thermodynamical criteria characterising Dopplershifts and line-intensity fluctuations of magnetic and non-magnetic features have been proven by observations.

Conclusions. We could account for differential Zeeman effects along the $\mathrm{D}_{1}$ line profile by combining expansion of flux tubes with height, low gas pressure inside flux tubes, and small filling factor due to seeing effects. Better agreement with observations, in particular with respect to magnetic field amplitudes, will probably need 3D models that take velocity fields and horizontal gradients of temperature into account.
\end{abstract}

Key words. techniques: spectroscopic - techniques: polarimetric - Sun: atmosphere - Sun: magnetic fields

\section{Introduction}

Many theoretical works have been devoted so far to solar magnetic flux tubes that have been concentrated by convective motions and are expanding into canopies (see for example Solanki et al. 1999; Uitenbroek 2003; Shelyag et al. 2004; Vögler et al. 2005; Okunev \& Kneer 2005). The comparison with observations is very difficult, because observational constraints can only be derived from a full 3D analysis of very fine structures, so that both accurate spectro-polarimetry and high spatial resolution are needed simultaneously.

The vertical structure of magnetic fields was investigated in particular by Eibe et al. (2002), Malherbe et al. (2004), and Roudier et al. (2006) in the sodium $\mathrm{D}_{1}$ line. While magnetic fields are decreasing with height in large spots, the situation appears to be different in faculae, where increasing fields are also observed. The same kind of result was obtained by Leka \& Metcalf (2003) with vector magnetic fields. The magnetic flux decreases with height in umbrae and penumbrae, but positive gradients are present in plages. This behaviour is one of the main topics of the present study.

The "Multichannel Subtractive Double Pass" mode of THEMIS provides accurate spectro-polarimetry of strong lines, without degrading the solar image by any slit-width. Moreover, strong lines, such as the sodium D lines, currently observed with this instrument, can provide measurements simultaneously at different levels of the solar atmosphere, by exploring different points along the profile. Deriving vertical gradients from only one line profile may also minimise the errors due to superposition of different wavelength ranges, for example in the case of differential atmospheric refraction or departures in the enlargement coefficients of the spectrograph.

In this paper, we use THEMIS/MSDP data of $589.6 \mathrm{~nm} \mathrm{NaI}$ to compare flux tubes observations with synthetic line profiles derived from magnetic models. Pure thermodynamical parameters (intensities and dopplershifts) are also investigated in the comparison of magnetic and non-magnetic features.

\section{Observations and processing of MSDP data}

The MSDP instrument of THEMIS is described in Mein (2002). Additional information can be found at http://www. themis.iac.es. The data of this paper were already used in Eibe et al. (2002). Spectro-polarimetric $I \pm V$ measurements were obtained on May 9, 2000, for the active region NOAA 8989 close to disk centre (E18, N17). The pixel size is approximately 0.2 arcsec.

The processing code is the standard MSDP code available in the data base http://bass2000.bagn.obs-mip.fr. On each point of the Sun, the $\mathrm{D}_{1}$-profile is determined by 16 points, with the wavelength step $0.008 \mathrm{~nm}$ (local bandwidth $0.004 \mathrm{~nm}$ ) The profile is smoothed by weights $1 / 4,1 / 2,1 / 4$ to reduce small discrepancies between even and odd channels, which correspond 

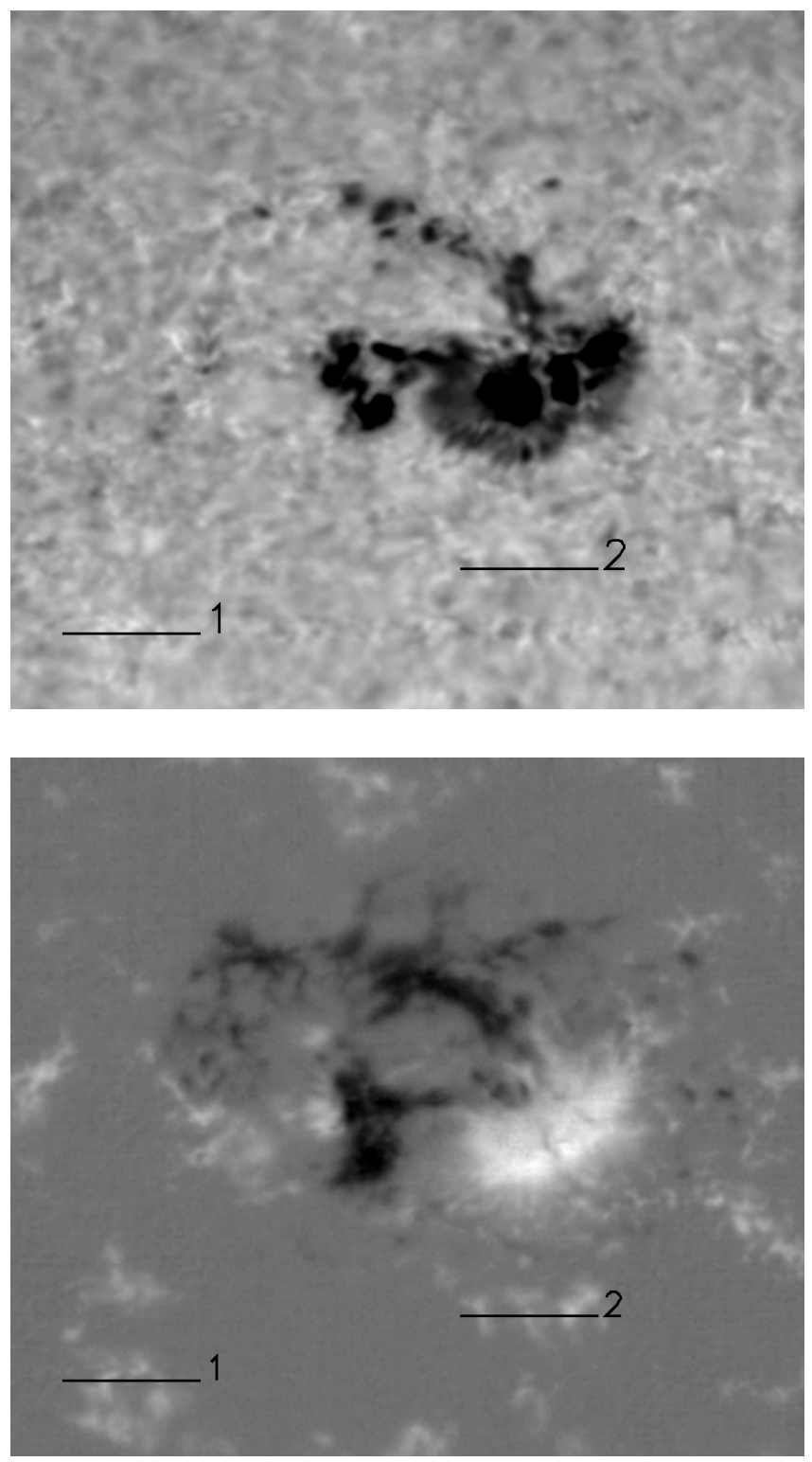

Fig. 1. Maps of intensity (top) and LOS magnetic field (bottom) at $\Delta \lambda=$ $0.024 \mathrm{~nm}$, with cuts (1) and (2). The field of view is $138^{\prime \prime} \times 121^{\prime \prime}$. North magnetic fields are white.

to different optical paths. Subsequent synthetic profiles take this smoothing into account.

Zeeman shifts, Doppler shifts, and intensity fluctuations are computed at each point of the $2 \mathrm{D}$-field by the so-called bisector method. They are determined by middle points of chords joining blue and red points of the profile, at given distances $2 \times \Delta \lambda$ (see Berlicki et al., 2006, Fig. 3). Line-of-sight magnetic fields are deduced from Zeeman shifts as in Eibe et al. (2002). A Zeeman shift of $1 \mathrm{G}$ is roughly equivalent to a Doppler shift of $0.9 \mathrm{~ms}^{-1}$ for the sodium $\mathrm{D}_{1}$ line. The results are given for 4 values of $\Delta \lambda: 0.008,0.016,0.024,0.032 \mathrm{~nm}$. Although successive values are not quite independent because of the smoothing, departures between extreme values are quite significant.

As an example, Fig. 1 shows the magnetic map obtained at $\Delta \lambda=0.024 \mathrm{~nm}$. Two cuts of 24 arcsec were selected. They are parallel to the lines of constant wavelength inside the MSDP channels. This minimises the errors due to line-profile interpolations between channels, along each cut. Figure 2 shows the line-of-sight (LOS) magnetic fields in Gauss, Fig. 3 the relative
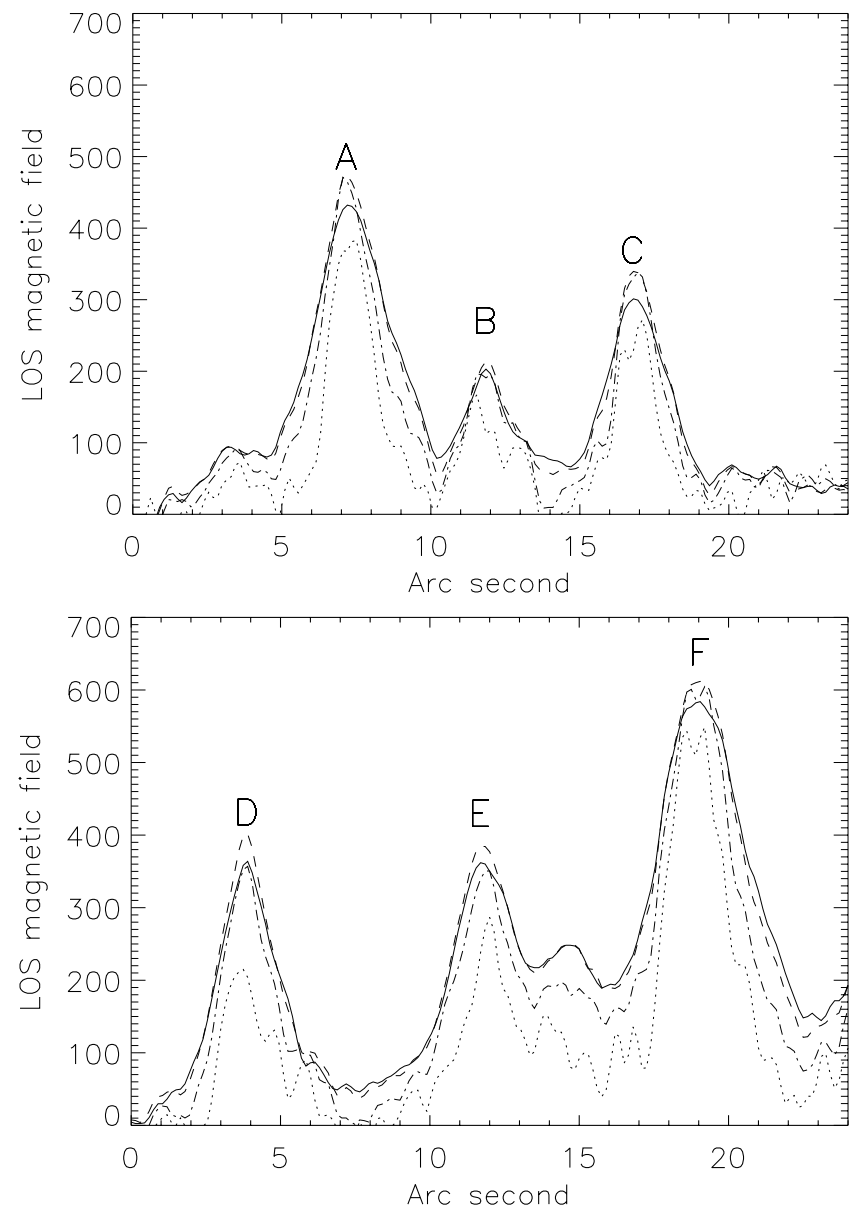

Fig. 2. LOS magnetic field along the cuts 1 (top) and 2 (bottom) for $\Delta \lambda=0.008 \mathrm{~nm}$ (solid lines), $0.016 \mathrm{~nm}$ (dashed), $0.024 \mathrm{~nm}$ (dasheddotted), $0.032 \mathrm{~nm}$ (dotted).

intensity fluctuations, and Fig. 4 the LOS velocities (positive = downward), deduced from the bisector method along cuts (1) and (2). All curves of Figs. 2-4 are referred to zero-values determined by nearby quiet solar regions. We call "line core" and "line wing" the data corresponding respectively to 0.008 and $0.032 \mathrm{~nm}$. We shall see later that the corresponding formation altitudes for Zeeman shifts differ roughly from $200 \mathrm{~km}$ (Fig. 8).

We analyse some magnetic and bright features in more detail. As indicated in the figures, we select from Fig. 2 six magnetic features ("flux tubes") $A, B, C, D, E, F$. Their half width at half maximum $(H W H M)$ is around 1 arcsec. They also correspond to maxima of intensities (Fig. 3) and generally to downward velocities (Fig. 4). From maxima of line-wing intensities at $0.032 \mathrm{~nm}$ in Fig. 3, we select five additional bright features $a, b, c, d, e, f$. They correspond to minima (upward velocities) in Fig. 4.

This small number of features cannot lead to a statistical study; however, we think that general trends can be extracted from them.

\section{Thermodynamical criteria of magnetic features}

In Fig. 5 we plot the wing-velocity (dotted lines of Fig. 4) versus the differences between wing-intensity and core-intensity fluctuations (dotted and solid lines of Fig. 3), deduced from peak-values of Figs. 3 and 4. We see that pure thermodynamical quantities, intensities and dopplershifts, allow magnetic and 

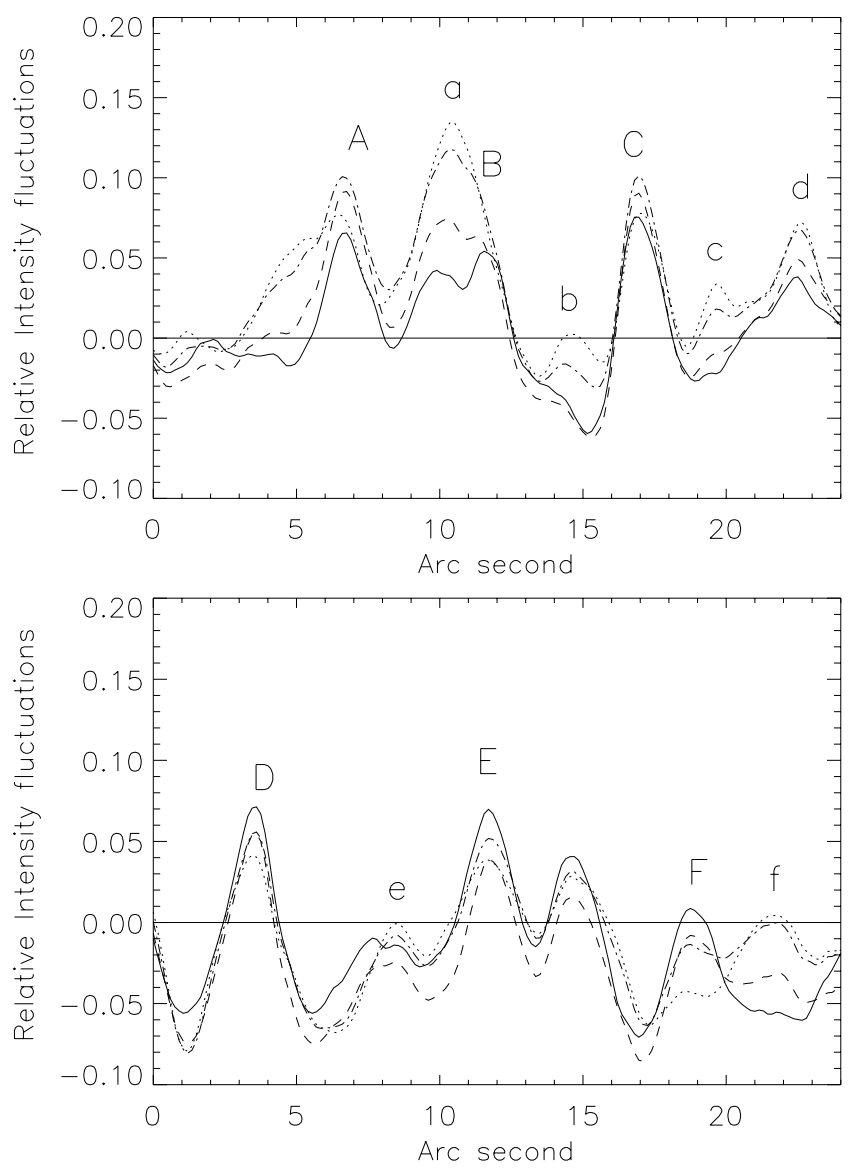

Fig. 3. Relative intensity fluctuations, referred to the mean intensity of a quiet area, along the cuts 1 (top) and 2 (bottom), for the same $\Delta \lambda$-values as in Fig. 2.

non-magnetic features to be distinguished, at least for our selected data. We can draw two conclusions:

- Magnetic features $A, C, D, E, F$, are characterised by downward velocities, while non-magnetic features $a, b, c, d, e$ are characterised by upward velocities (the opposite cases of $B$ and $f$ are probably due to blends with $a$ and $F$, respectively). This was expected, since downward velocities of magnetic flux tubes are well known (see for example Malherbe et al. 2004; Rimmele 2004), while pure convective motions mean ascending high-temperature material.

- The relative wing-intensity minus the relative core-intensity is smaller for magnetic features $A, B, C, D, E, F$ than for non-magnetic features $a, b, c, d, e, f$. This was also expected. Convective motions are decreasing in the upper levels of the atmosphere. Corresponding temperature fluctuations are higher in the lower levels, leading to high wing-intensity fluctuations.

\section{Mean magnetic feature}

Let us concentrate now on the LOS magnetic fields shown in Fig. 2. We see that line-wing magnetic fields are generally smaller than line-core magnetic fields. Moreover, the spatial width of magnetic features $A, B, C, D, E, F$ is smaller in the wings than in the core of the line. To illustrate this point and to prepare easier comparison with synthetic profiles, we define an average magnetic feature. In each case of $A, B, C, D, E, F$ structures and for each value of $\Delta \lambda$, we determine the abscissa $x_{0}$
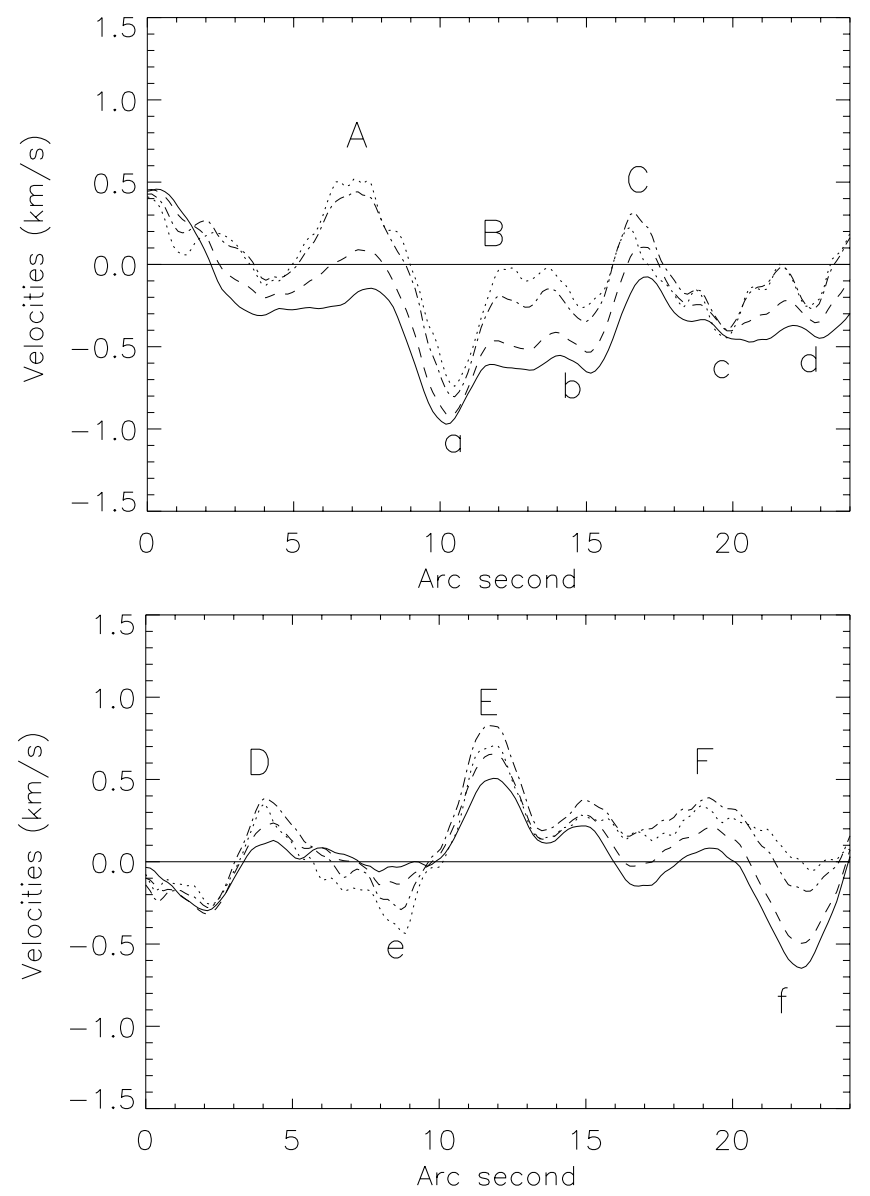

Fig. 4. LOS velocities along the cuts 1 (top) and 2 (bottom), for the same $\Delta \lambda$-values as in Fig. 2 (positive $=$ downward).

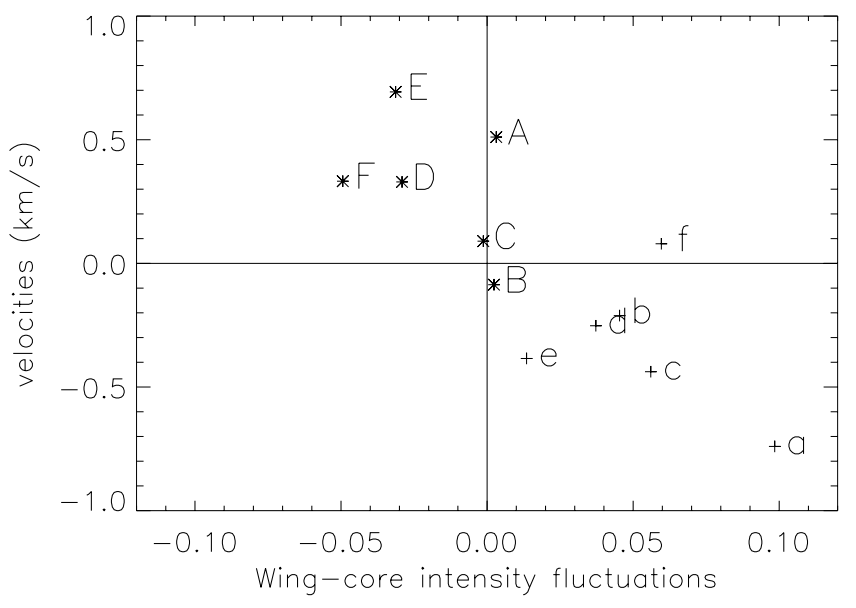

Fig. 5. Wing velocity (at $0.032 \mathrm{~nm}$ ) versus difference between intensity fluctuations in line wing $(0.032 \mathrm{~nm})$ and line core $(0.008 \mathrm{~nm})$ for magnetic features $A, B, C, D, E, F$ and non-magnetic features $a, b, c, d, e, f$.

of the maximum magnetic field. We call $B_{-}(\Delta \lambda, l)$ the magnetic field at the point $x_{0}-l$ and $B_{+}(\Delta \lambda, l)$, the magnetic field at $x_{0}+l$. Figure 6 shows, for each $\Delta \lambda$, the functions of $l$ defined by

$B(\Delta \lambda, l)=<B_{-}(\Delta \lambda, l)+B_{+}(\Delta \lambda, l)>/ 2$

where the average values are taken over $A, B, C, D, E, F$. They characterise a symmetrized mean feature that can be compared to synthetic calculations. Crosses are plotted at points where

$B(\Delta \lambda, l)=B(\Delta \lambda, 0) / 2$.

They define the $H W H M W(\Delta \lambda)$. 


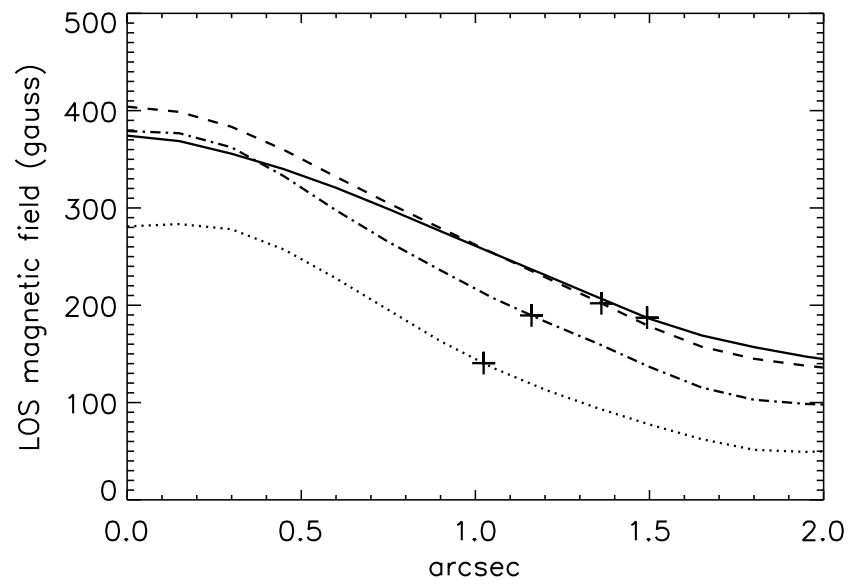

Fig. 6. Average observed magnetic feature: LOS magnetic field as a function of the distance to the axis of the symmetrized feature for $\Delta \lambda=0.008 \mathrm{~nm}$ (solid lines), $0.016 \mathrm{~nm}$ (dashed), $0.024 \mathrm{~nm}$ (dasheddotted), $0.032 \mathrm{~nm}$ (dotted). Crosses show the points of half maximum values for each curve.

\section{Is the $D_{1}$-line formed inside canopy layers?}

In Fig. 6, we see that the widths $W(\Delta \lambda)$ increase from line wing to line core:

$W(0.032)<W(0.024)<W(0.016)<W(0.008)$.

This agrees with the observations mentioned by Eibe et al. (2002), Leka \& Metcalf (2003), and Malherbe et al. (2004). It suggests that we observe flux tubes expanding into canopies between the wings and the core of the $\mathrm{D}_{1}$-line. But on the axis of the feature, we observe that

$B(0.032,0)<B(0.008,0)<B(0.024,0)<B(0.016,0)$.

Since the LOS magnetic flux across a given flux tube is expected to be the same at all levels of the solar atmosphere, we should expect that, for a given $\Delta \lambda$ value, the integrals of $B(\Delta \lambda, l)$ over $l$ are independent of $\Delta \lambda$. High $B$-values close to the axis should compensate low $B$-values far from the axis. This is not the case, in particular for $\Delta \lambda=0.032 \mathrm{~nm}$. We must go further into synthetic profile calculations to try to understand such a discrepancy.

\section{A 2D model flux tube (I) compensating horizontal components of Lorentz forces}

\subsection{Magnetic field and gas pressure}

Our THEMIS observations, as well as G-band observations of filigrees (see e.g. Shelyag et al., 2004), show that many elongated structures may be relevant to $2 \mathrm{D}$ structures. We shall consider a 2D flux tube model with vertical axis in the $z$-direction, but not consider 3D models. We made some tests that pointed out that they lead qualitatively to the same kind of results.

The altitudes are referred to the level $\tau_{5000}=1$. We define the vertical component of magnetic field along the symmetry axis by

$B_{z}(0, z)=B_{z}(0,0) \cdot \exp (-z / h)$.

At each altitude $z$, the horizontal variation of $B_{z}$ inside the tube is defined by

$$
\begin{array}{r}
B_{z}(x, z)=B_{z}(0, z) \cdot \cos ^{2}(\pi x /(4 d(z)) \\
-1<x / 2 d(z)<1 .
\end{array}
$$

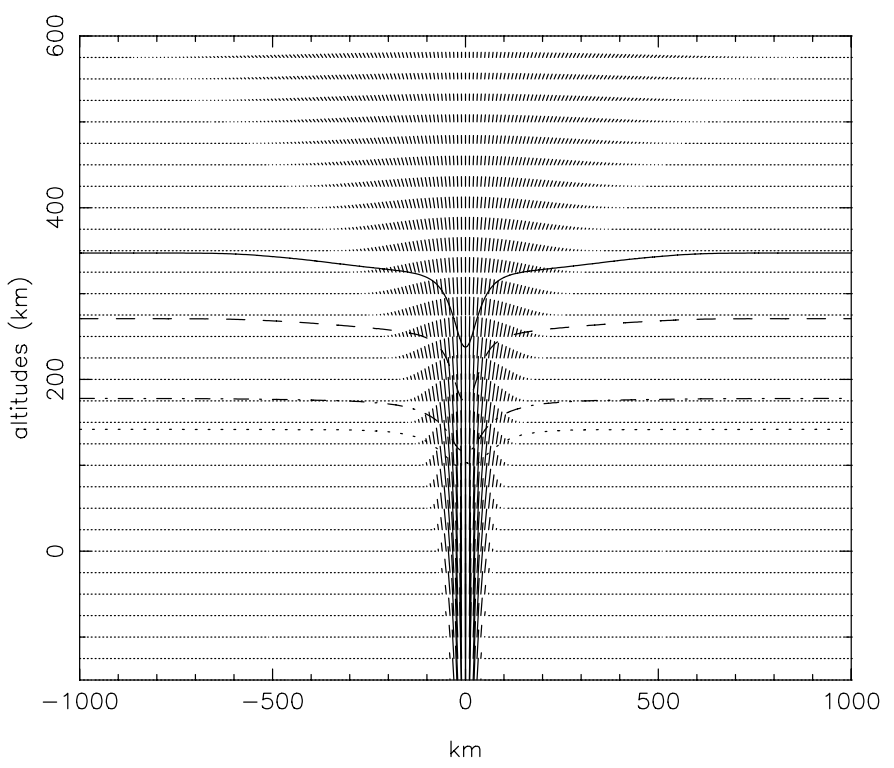

Fig. 7. 2D model magnetic field (I). Formation altitudes corresponding to $\Delta \lambda=0.008,0.016,0.024$, and $0.032 \mathrm{~nm}$ are plotted repectively with solid, dashed, dashed-dotted, and dotted lines.

To keep a constant flux versus $z$, we assume

$d(z)=d(0) \cdot B_{z}(0,0) / B_{z}(0, z)$

where $d(0)$ is the $H W H M$ of the tube, at the level $\tau_{5000}=1$.

The horizontal magnetic field component is defined by the zero-divergence

$\frac{\partial B_{x}(x, z)}{\partial x}=-\frac{\partial B_{z}(x, z)}{\partial z}$

with

$B_{x}(-\infty, z)=0$

We use a quiet solar model outside the flux tube. Inside the tube, we keep the same temperature at the same altitude and modify all densities so that the total plasma pressure $P(x, z)$ (including the turbulent pressure) compensates for the horizontal component of the Lorentz force

$\frac{\partial P(x, z)}{\partial x}=\frac{B_{z}(x, z)}{\mu_{0}}\left(\frac{\partial B_{x}(x, z)}{\partial z}-\frac{\partial B_{z}(x, z)}{\partial x}\right)$.

Figure 7 shows the magnetic field model (I) obtained with

$B_{z}(0,0)=1100 \mathrm{G}$

$h=240 \mathrm{~km}$

$d(0)=40 \mathrm{~km}$.

The width at half maximum $2 d(0)$ assigned to the flux tube at the zero altitude is close to 0.1 arcsec. Let us note that it is smaller than the width at the altitudes of line formation. Magnetic vectors are plotted by dashes, the length of which are proportional to the magnetic field amplitude. Note that some anamorphosis occurs in the figure because of different scales in $x$ and $z$ coordinates. 


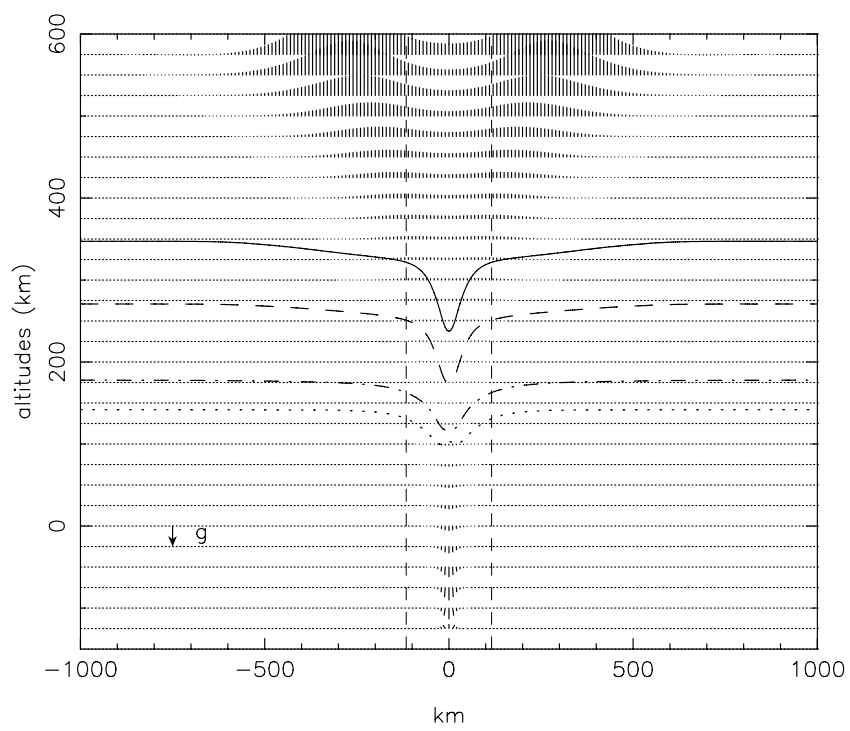

Fig. 8. Acceleration vectors showing the departures from magnetostatic equilibrium of model (I). The vector $g$ specifies the solar gravity. Formation altitudes corresponding to $\Delta \lambda=0.008,0.016,0.024$, and $0.032 \mathrm{~nm}$ are plotted repectively with solid, dashed, dashed-dotted, and dotted lines. Vertical dashed lines show the range defined by $-2 W_{w}(0.008)<x<2 W_{w}(0.008)$ (see the text, Sect. 7).

\subsection{Departures from equilibrium}

The total plasma pressure inside our model satisfies Eq. (11), which ensures the horizontal equilibrium. But the vertical component of the Lorentz force is not compensated everywhere. Figure 8 shows the acceleration vectors derived from the equations

$$
\begin{aligned}
\rho \gamma_{x}= & -\frac{\partial P(x, z)}{\partial x} \\
& +\frac{B_{z}(x, z)}{\mu_{0}}\left(\frac{\partial B_{x}(x, z)}{\partial z}-\frac{\partial B_{z}(x, z)}{\partial x}\right) \\
\rho \gamma_{z}= & -\frac{\partial P(x, z)}{\partial z} \\
& -\frac{B_{x}(x, z)}{\mu_{0}}\left(\frac{\partial B_{x}(x, z)}{\partial z}-\frac{\partial B_{z}(x, z)}{\partial x}\right)-\rho g
\end{aligned}
$$

where $\rho$ is the plasma density, $\gamma_{x}$ and $\gamma_{z}$ the components of the acceleration vector, and $g$ the solar gravity.

Since Eq. (11) is satisfied, the vectors are vertical. On the tube axis, the acceleration is zero in the middle part of the plot, where the scale-height $h$ of the magnetic field is adjusted to twice the density scale height of the VAL3C model. Small upward and downward accelerations are present at the top and the bottom, where the scale height is not adjusted perfectly.

On both edges of the tube, upward accelerations appear at high altitudes, mainly because of the decrease in density resulting from Eq. (11). We discuss in Sect. 7 the $(x, z)$ range responsible for line profile observations and magnetic field measurements. In Sect. 9 we propose an alternative model with smaller departures from equilibrium.

\section{Synthetic $\mathrm{NaD}_{1}$ spectra for model (I)}

\subsection{NLTE line profiles}

We use the VAL3C model atmosphere as the quiet reference outside the flux tube (Vernazza et al. 1981). The $\mathrm{NaD}_{1}$ profiles

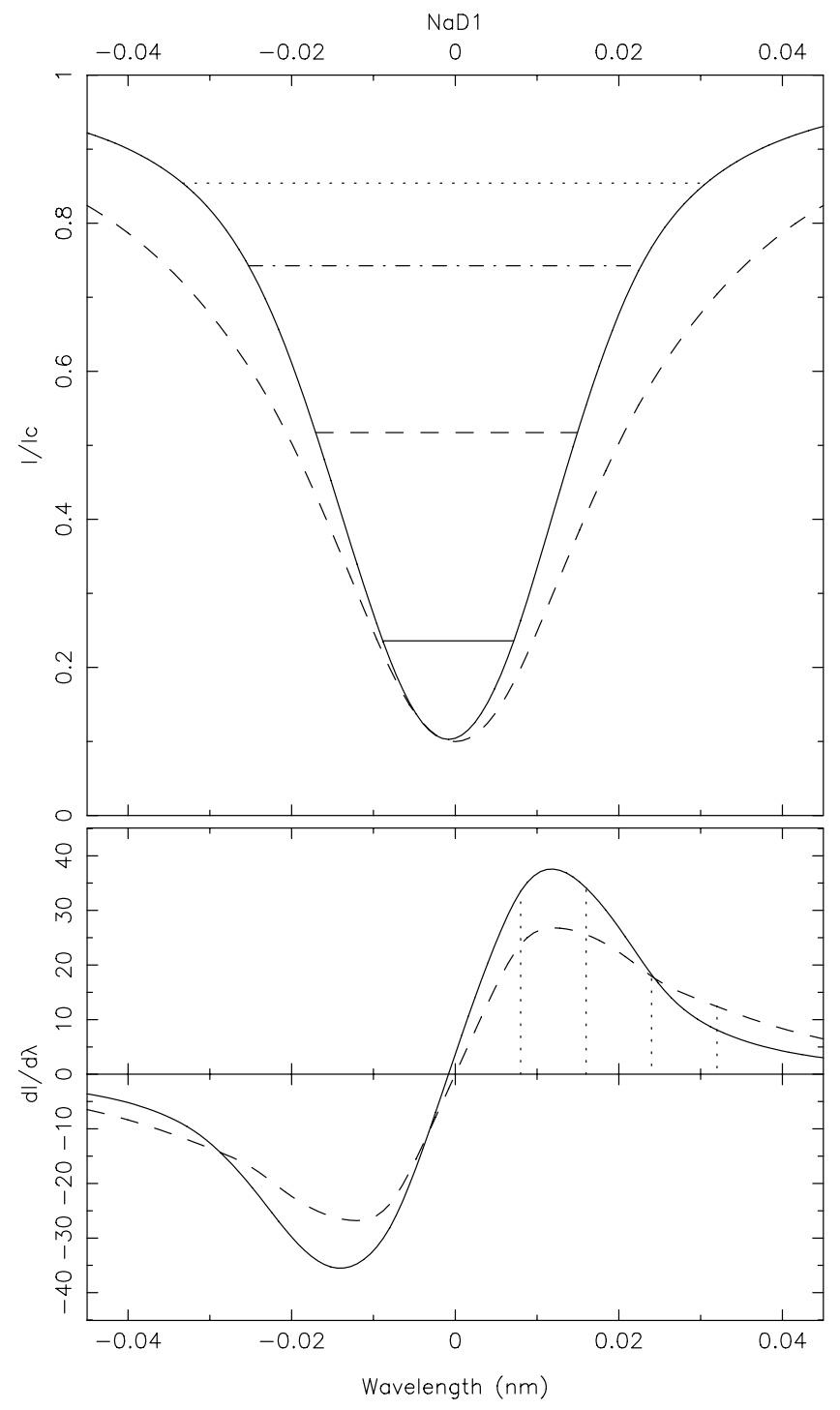

Fig. 9. Synthetic Stokes (I-V) $\mathrm{NaD}_{1}$ profiles (top) and slopes $\partial I / \partial \lambda$ (bottom). Solid line: centre of the flux tube, dashed line: quiet profile outside flux tube. The chords used in the bisector method are plotted in the upper diagram: solid, dashed, dashed-dotted, and dotted lines correspond to $\Delta \lambda=0.008,0.016,0.024$, and $0.032 \mathrm{~nm}$, respectively.

are deduced from the 2.2 version of the NLTE radiative transfer code MULTI (Carlsson 1986). They are smoothed so as to mimic the profiles deduced from MSDP observations. Since the data are not observed far from disk centre (E18, N17), we assume that calculations at disk centre $(\mu=1)$ provide a good qualitative result. We restrict the model to altitudes smaller than $600 \mathrm{~km}$, assuming that the contribution of higher layers to the $\mathrm{NaD}_{1}$ line can be neglected. In the low photosphere, we extrapolate the model down to $-150 \mathrm{~km}$, to get a reasonable optical depth along the flux tube axis, where Lorentz forces imply reducing the matter density. We use the assumption of weak magnetic field, so that, at each altitude, the absorption coefficient in the line is translated by the corresponding Zeeman shift for both Stokes profiles $(I+V)$ and $(I-V)$.

The upper Fig. 9 shows the synthetic profile $(I-V)$ corresponding to the axis of the flux tube, with the 4 chords corresponding to the 4 wavelengths used in Sect. 2 specifying the LOS magnetic field through the solar atmosphere by the bisector method. The $(I-V)$ profile is blue-shifted in the tube axis 


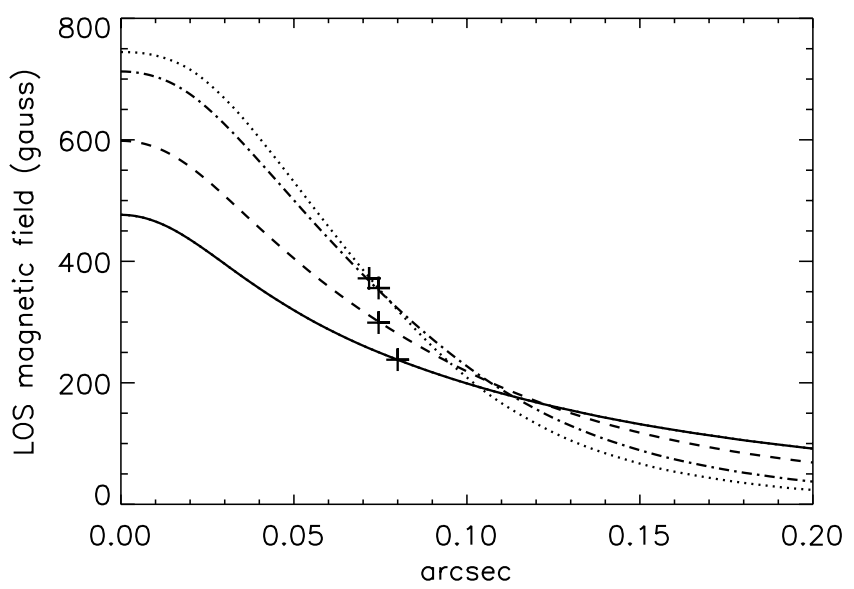

Fig. 10. Synthetic LOS magnetic fields deduced from the flux tube model (I). As in Fig. 2, solid, dashed, dashed-dotted, and dotted lines correspond to $\Delta \lambda=0.008,0.016,0.024$, and $0.032 \mathrm{~nm}$, respectively. Crosses show the points of half maximum values at $W(\Delta \lambda)$ from the tube axis.

because the magnetic field is upward (like Doppler profiles with upward velocities).

To visualise formation heights of the 4 wavelengths used in Sect. 2, we introduce small magnetic field fluctuations, linear versus altitude, and directly determine the altitudes where the fluctuations correspond to the synthetic Zeeman shifts. This is equivalent to the weighting-function or response-function method (Mein 1971; Beckers et al. 1975) used for the sodium lines by Eibe et al. (2001, 2002). The results are plotted in Figs. 7 and 8. Dips in the formation altitudes inside the flux tube are due to the density decrease resulting from the compensation of the horizontal component of Lorentz forces.

\subsection{Synthetic magnetic field across the flux tube}

Zeeman shifts are calculated for all Stokes $(I-V)$ profiles deduced from the disturbed atmospheres across the flux tube. Figure 10 shows the obtained LOS magnetic fields. Two results agree with average observations displayed in Fig. 6. The halfwidths of the curves at different $\Delta \lambda$ values satisfy Eq. (3), which reflects the expansion of magnetic structures with height, and the order of magnitude of LOS magnetic field close to the axis is similar to the observed ones.

But the LOS magnetic fields at $\Delta \lambda=0.032 \mathrm{~nm}$ exhibit the highest values in the centre of the flux tube, while observations show the lowest ones. The Eq. (4) is no longer valid. Moreover, the synthetic widths $W(\Delta \lambda)$ are too small by a factor around 10 .

We now try to improve the agreement by introducing seeing effects.

\subsection{Seeing effects}

To simulate seeing effects, we convolve the distribution, along the $\mathrm{x}$-axis, of the synthetic Stokes $(I-V)$ intensities with the kernel

$S(x)=\cos ^{2}(\pi x / 4 s) \quad-1<x / 2 s<1$

for all wavelengths.

Let us call $B_{s}(\Delta \lambda, 0)$ the magnetic fields close to the centre of the flux tube and $W_{s}(\Delta \lambda)$ the widths of the magnetic structure, corresponding to seeing-effect calculations. Figure 11 shows the

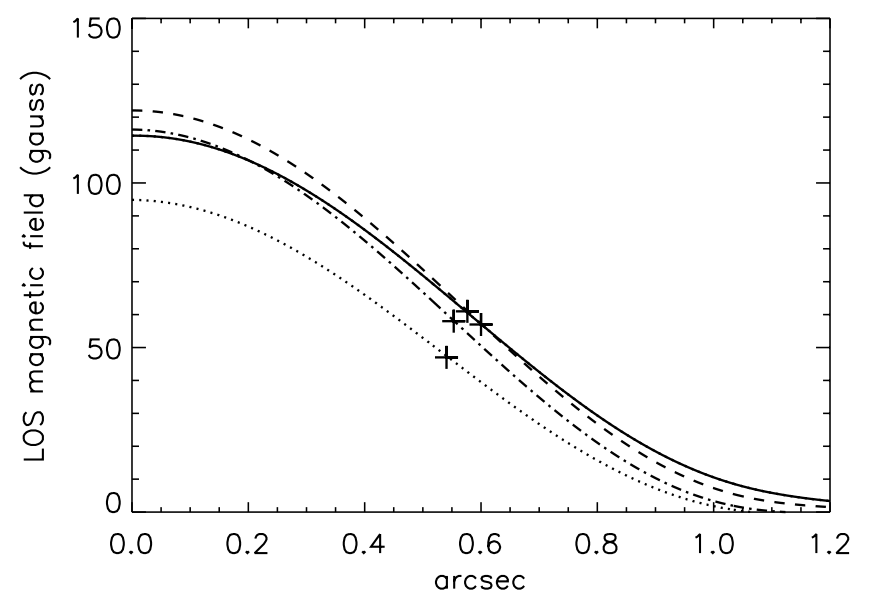

Fig. 11. Synthetic LOS magnetic fields deduced from the flux tube model (I), with seeing effects $(s=400 \mathrm{~km}) . \Delta \lambda$ values are the same as in Fig. 10. Crosses show the points of half maximum values at $W_{s}(\Delta \lambda)$ from tube axis.

result in the case of $s=400 \mathrm{~km}$. Both observational relationships found in Sect. 5 are now satisfied:

$W_{s}(0.032)<W_{s}(0.024)<W_{s}(0.016)<W_{s}(0.008)$

$B_{s}(0.032,0)<B_{s}(0.008,0)<B_{s}(0.024,0)<B_{s}(0.016,0)$.

But the order of magnitude of $W_{s}$-values is too low by a factor 2 , and the LOS magnetic field values, close to the axis, are too low, by a factor 4 .

In conclusion, seeing effects lead to better qualitative agreement, although widths and field-amplitudes are not large enough. We discuss in Sect. 10 the limitations of our models in this respect.

\subsection{Departures from equilibrium and magnetic field measurements}

Subsequently, we call $W_{w}$ the synthetic widths corresponding to calculations without seeing effects. The highest values of $W_{w}$ corresponds to $0.008 \mathrm{~nm}$. The strong decrease in magnetic field between $x=0$ and $x=2 W_{w}(0.008)$ shows that photons coming from outside this range do not affect the magnetic fields of Fig. 11 very much, although the convolution by seeing effects operates over a wider range. We have plotted the range $-2 W_{w}(0.008)<x<2 W_{w}(0.008)$ in Fig. 8. The regions of a large departure from equilibrium lie outside this range, at high altitudes on both sides. This means that magnetic field observations depend very little on regions out of equilibrium.

\section{Discussion}

\subsection{Size of magnetic features}

Equation (3), which states that widths $W$ are increasing from line wings to line core, holds for all Figs. 6, 10, and 11. Figure 7 shows the flux tube expansion with height. It also shows that the Zeeman shifts in the line core are formed between 150 and $200 \mathrm{~km}$ above the Zeeman shifts in line wings. Although our calculations of formation heights (Sect. 7.1) are strictly valid only in the case of small magnetic fields linear versus altitude, they qualitatively account for the increase in widths in Figs. 10 and 11 . 


\subsection{Seeing effects at flux tube centre}

Let us call $B_{w}(\Delta \lambda, x)$ and $B_{s}(\Delta \lambda, x)$ the synthetic LOS magnetic fields plotted in Fig. 10 (tube without seeing effects) and 11 (tube with seeing effects). In Fig. 10

$B_{w}(0.032,0)>B_{w}(0.008,0)$,

while Fig. 11 satisfies the equation

$B_{s}(0.032,0)<B_{s}(0.008,0)$,

like observations in Fig. 6. To understand this departure, let us consider a simpler case where the magnetic field should not depend on $x$ inside the flux tube, and where the seeing effects should be a convolution by a constant function. Let us call $w_{\Delta \lambda}$ the half-width of the tube at the formation altitude of $\Delta \lambda$, and $s$ the half-width of the seeing function. At the centre of the tube, the seeing effect is equivalent to the filling factor $w_{\Delta \lambda} / s$ between the intensity in the tube and the neighbouring quiet sun. Let us call $I_{w}(\Delta \lambda, 0), V_{w}(\Delta \lambda, 0)$ the Stokes parameters at flux tube centre without seeing effects, $I_{s}(\Delta \lambda, 0), V_{s}(\Delta \lambda, 0)$ the Stokes parameters at flux tube centre with seeing effects, and $I_{q}(\Delta \lambda, 0)$ the quiet sun intensity. We can write both equations as

$I_{s}(\Delta \lambda, 0)=I_{w}(\Delta \lambda, 0) w_{\Delta \lambda} / s+\left(1-w_{\Delta \lambda} / s\right) I_{q}(\Delta \lambda, 0)$

$V_{s}(\Delta \lambda, 0)=V_{w}(\Delta \lambda, 0) w_{\Delta \lambda} / s$.

Let us assume that the Zeeman shifts are much smaller than the width of the line profile, so that the slopes of the line profiles $\partial I_{s} / \partial \lambda, \partial I_{w} / \partial \lambda$, and $\partial I_{q} / \partial \lambda$ do not depend on the magnetic field and are symmetrical at both ends of the chords in the bisector analysis. The V-profiles are proportional to the measured magnetic field times the slope of the profile $\frac{\partial I}{\partial \lambda}$. Equation (20) can be written, at tube centre:

$B_{s}(\Delta \lambda, 0) \frac{\partial I_{s}}{\partial \lambda}=B_{w}(\Delta \lambda, 0) \frac{\partial I_{w}}{\partial \lambda} w_{\Delta \lambda} / s$

with the same notations as before for $B_{s}$ and $B_{w}$. By derivating Eq. (19), we can eliminate $\frac{\partial I_{s}}{\partial \lambda}$ :

$\frac{B_{w}(\Delta \lambda, 0)}{B_{s}(\Delta \lambda, 0)}=1+\alpha(\Delta \lambda, 0) \cdot\left(s / w_{\Delta \lambda}-1\right)$

with

$\alpha(\Delta \lambda, 0)=\frac{\partial I_{q}}{\partial \lambda} / \frac{\partial I_{w}}{\partial \lambda}$.

Equations (22) and (23) can be used qualitatively for our model of flux tubes, on condition that $\alpha(\Delta \lambda, 0)$ is replaced by some average of $\alpha(\Delta \lambda, x)$.

The lower Fig. 9 shows the slope $\partial I / \partial \lambda$ of line profiles from model (I) at flux tube centre $\left(\partial I_{w} / \partial \lambda\right.$, solid line) and far from the flux tube $\left(\partial I_{q} / \partial \lambda\right.$, dashed line), which corresponds to the quiet VAL3C model. It is easy to see that $\alpha(0.008,0)<1$, while $\alpha(0.032,0)>1$. This is due to the low opacity of the tube, combined with the vertical temperature gradient of the VAL3C model.

Figure 12 shows the variation in $\alpha(\Delta \lambda, x)$ versus $x$, for the 4 values of $\Delta \lambda$ used in Fig. 10 . The values at $x=0$ result directly from the lower plots of Fig. 9. At high values of $x, \alpha(\Delta \lambda, x)$ becomes close to 1 , whatever $\Delta \lambda$ is (quiet sun). We see in Fig. 12 that $\alpha(0.008, x)$ remains smaller than $\alpha(0.032, x)$ in the full range of $x$ where the magnetic field is large (Fig. 10). Moreover, the width $w_{\Delta \lambda}$ decreases when $\Delta \lambda$ increases. So, the term

$\alpha(\Delta \lambda, 0) \cdot\left(s / w_{\Delta \lambda}-1\right)$

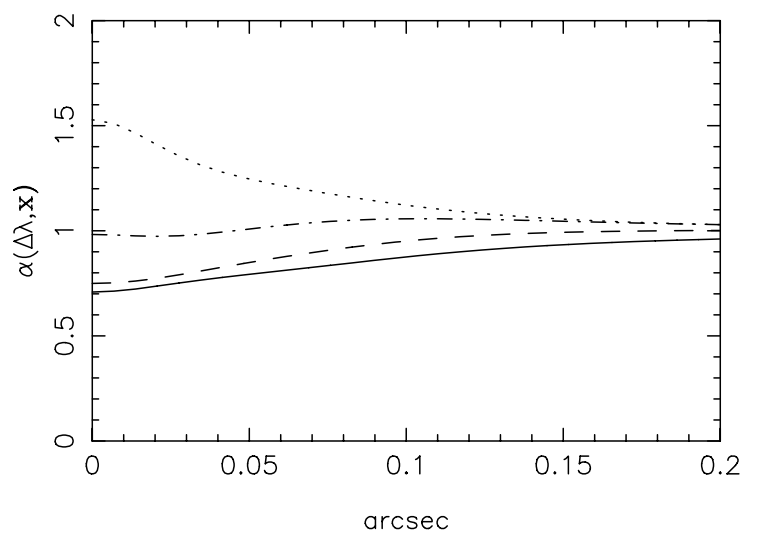

Fig. 12. Parameters $\alpha(\Delta \lambda, x)$ deduced from the slopes of line profiles in flux tube model (I) and Eq. (23). They characterise the weights of Zeeman shifts in the case of convolution by seeing effects (see the text). Solid, dashed, dashed-dotted, and dotted lines correspond to $\Delta \lambda=0.008,0.016,0.024$, and $0.032 \mathrm{~nm}$, respectively, from line-centre.

of Eq. (22) is smaller for $\Delta \lambda=0.008$ than for $\Delta \lambda=0.032$.

In conclusion, although $B_{w}(0.032,0)>B_{w}(0.008,0)$ (Fig. 10), both equations

$\alpha(0.032, x)>\alpha(0.008, x)$

$w_{0.032}<w_{0.008}$

can account for the relationship $B_{s}(0.032,0)<B_{s}(0.008,0)$ (Fig. 11).

\subsection{Integrated magnetic fluxes}

The discrepancy between integrated magnetic fluxes over the whole structure at different $\Delta \lambda$-values was already noted for observed data (Figs. 2 and 6). Since the width $s$ of the seeing function (Eq. (14)) is much larger than the width $d$ of the tube, it can be expected that the shape of $B_{s}(\Delta \lambda, x)$ versus $x$ looks like the seeing function itself. Indeed, the curves $B_{s}(0.008, x)$ and $B_{s}(0.032, x)$ of Fig. 11 are more similar than the curves $B_{w}(0.008, x)$ and $B_{w}(0.032, x)$ of Fig. 10. In particular, $B_{s}(0.032, x)$ is smaller than $B_{s}(0.008, x)$ for all $\mathrm{x}$-values. The result is that the magnetic flux integrated over the full observed structure reflects the same behaviour versus $\Delta \lambda$ as the magnetic field at flux tube centre (Eq. (18)).

\section{Model flux tube (II) closer to static equilibrium}

To try to reduce the amplitudes of accelerations characterising the departures from equilibrium (Fig. 8), we compute a new model flux tube (II). We keep Eqs. (6)-(10). But we replace Eq. (5), which defines the vertical component of the magnetic field along the tube axis, by the condition

$B_{z}(0, z)^{2} / 2 \mu_{0}=R \cdot P(-\infty, z)$

where the ratio $R$ does not depend on $z$, and where $P(-\infty, z)$ is the pressure of the quiet sun model. $B_{z}(x, z)$ is still defined by Eq. (6). But Eq. (11), which specifies $P(x, z)$, is replaced by

$P(x, z)=P(-\infty, z)-B_{z}(x, z)^{2} / 2 \mu_{0}$.

In this way, the gas pressure is never reduced to zero, and model (II) might be extended at very high altitudes, contrary to model (I). 


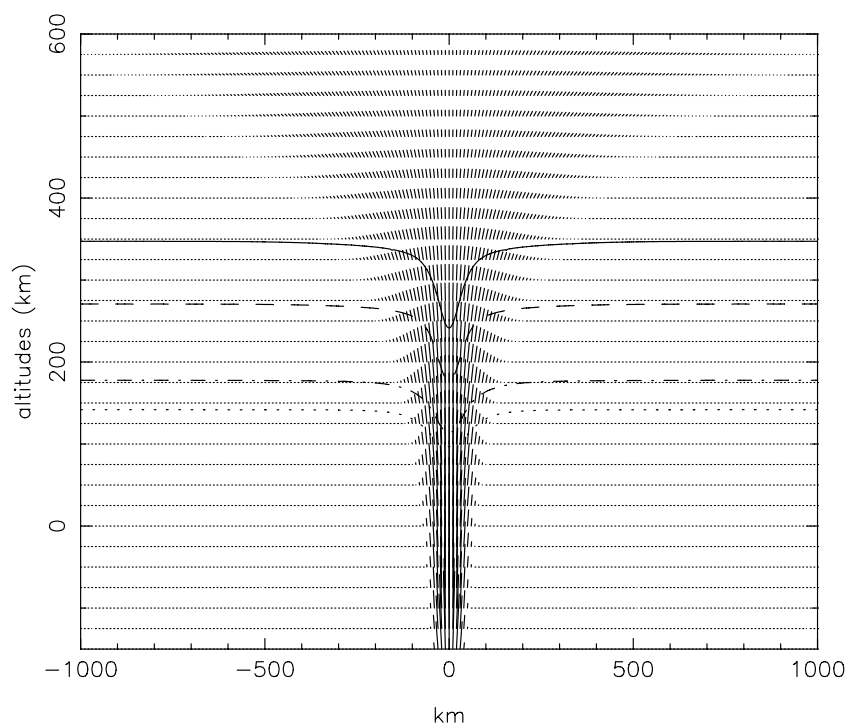

Fig. 13. Magnetic field as in Fig. 7, but for model (II).

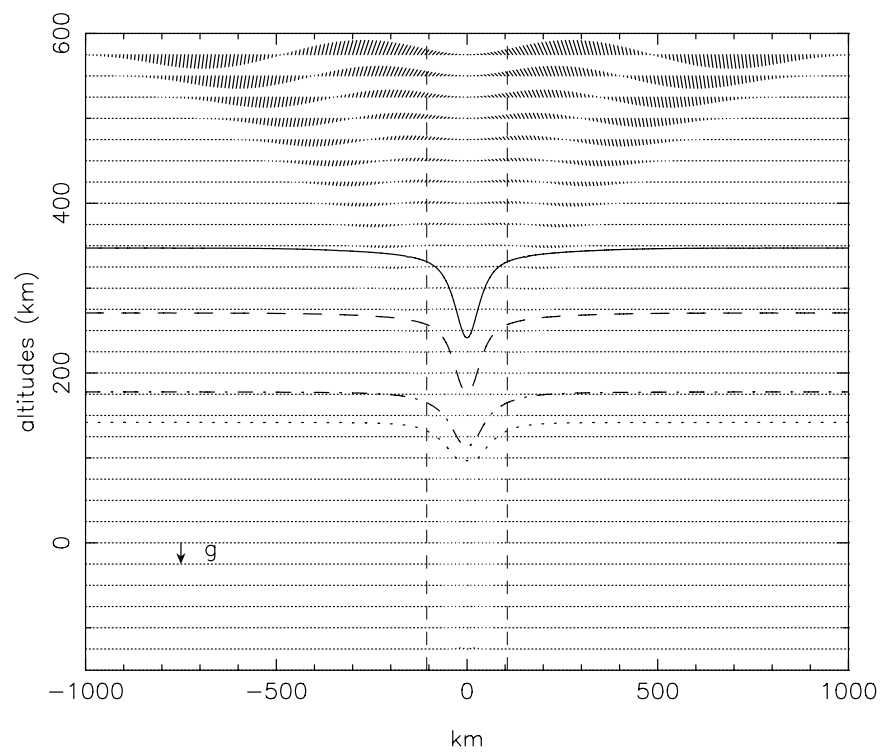

Fig. 14. Acceleration vectors showing the departures from magnetostatic equilibrium as in Fig. 8, but for model (II).

Figure 13 shows the magnetic field model (II) with

$R=0.4$

$d(0)=40 \mathrm{~km}$.

In Fig. 14 the resulting departures from equilibrium are plotted. By comparison with Fig. 8, we see that the equilibrium is better inside the range $-2 W(0.008)<x<2 W(0.008)$. Outside this range, the accelerations are no longer vertical, but the amplitudes are always smaller than the solar gravity. We also note that the formation altitudes are less depressed outside the range $-2 W(0.008)<x<2 W(0.008)$ than in Fig. 8. This is due to the fact that the compensation of horizontal components of Lorentz forces in Fig. 8 evacuated the edges of the tube more and more at high altitudes.

The Fig. 15 displays the synthetic LOS magnetic field of model (II) with seeing convolution $s=500 \mathrm{~km}$. It is very similar to Fig. 11, and shows that the results do not depend very much on

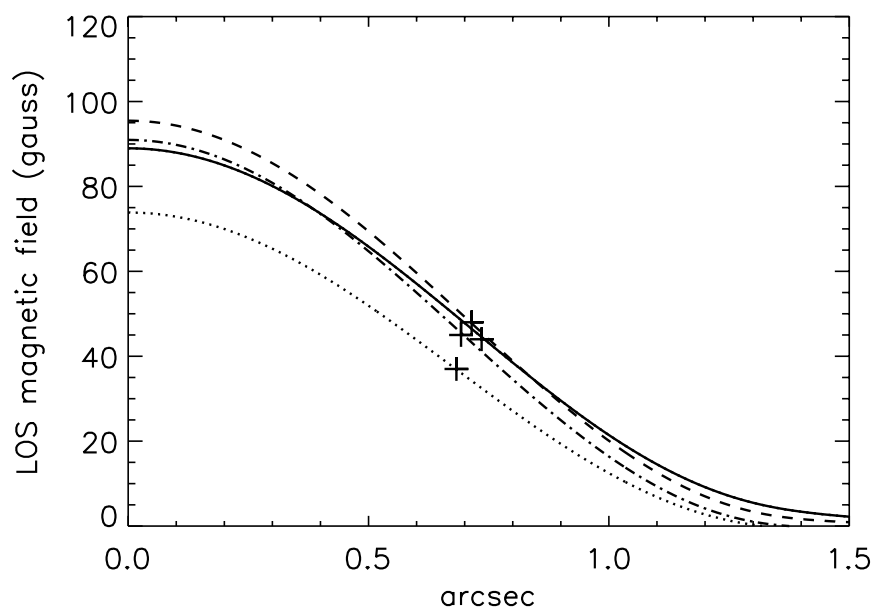

Fig. 15. Synthetic LOS magnetic fields deduced from the flux tube model (II), with seeing effects $(s=500 \mathrm{~km}) . \Delta \lambda$ values are the same as in Fig. 11. Crosses show the points of half maximum values at $W_{s}(\Delta \lambda)$ from tube axis.

the kind of Lorentz-force compensation. In particular, Eqs. (15) and (16) hold as much for model (II) as for model (I).

Let us note, however, that other models similar to model (II) would satisfy both equations, on condition that $\mathrm{R}$ and $\mathrm{s}$ are selected suitably. For example, $R=0.5$ and $s=300 \mathrm{~km}$ also lead to a possible solution, with higher departures from equilibrium, lower widths $W$, and higher magnetic field measurements along tube axis.

\section{Model (III): conglomerate of inclined flux tubes}

Models (I) and (II) reproduce the observations qualitatively but not quantitatively. A better agreement should imply larger synthetic magnetic fields $\mathrm{B}_{s}$ and larger widths $\mathrm{W}_{s}$. Model (I) is not able to accept any increase of magnetic field, because the pressure reduction at high levels cannot exceed the pressure itself. In contrast, model (II) can be used with increased values of the width $d(0)$ of the flux tube, and consequently increased values of the field, after seeing convolution. But any increase in $d(0)$ also increases the departures from equilibrium, and accelerations of Fig. 14 become drastically larger than solar gravity.

Let us note that the multiple peaks observed in Fig. 2 at $0.032 \mathrm{~nm}$ suggest flux tubes merging between formation heights of line wings and line core of $\mathrm{NaD}_{1}$. This may suggest conglomerates of flux tubes of the same polarity, as proposed by Okunev $\&$ Kneer (2005). But a new difficulty arises with tubes similar to model (II). At high levels, where tubes are merging, the total field intensity exceeds the limit implied from gas pressure. We can get round this difficulty by inclining flux tubes.

We propose a model (III) that is the sum of 5 tubes. The tube axis are straight lines. The central tube is vertical. At altitude zero, the widths of flux tubes are defined by $d(0)=20 \mathrm{~km}$, and the horizontal distance between successive axes is $150 \mathrm{~km}$. From one tube to the next, the inclination $\gamma=\partial x / \partial z$ increases by 0.6 , so that the angles with the vertical direction are roughly 31 and 50 degrees. For each tube, we again keep Eqs. (6)-(10), but we replace Eq. (26) by

$B_{z}\left(x_{0}, z\right)^{2} \cdot\left(1+\gamma^{2}\right) / 2 \mu_{0}=R \cdot P(-\infty, z)$ 


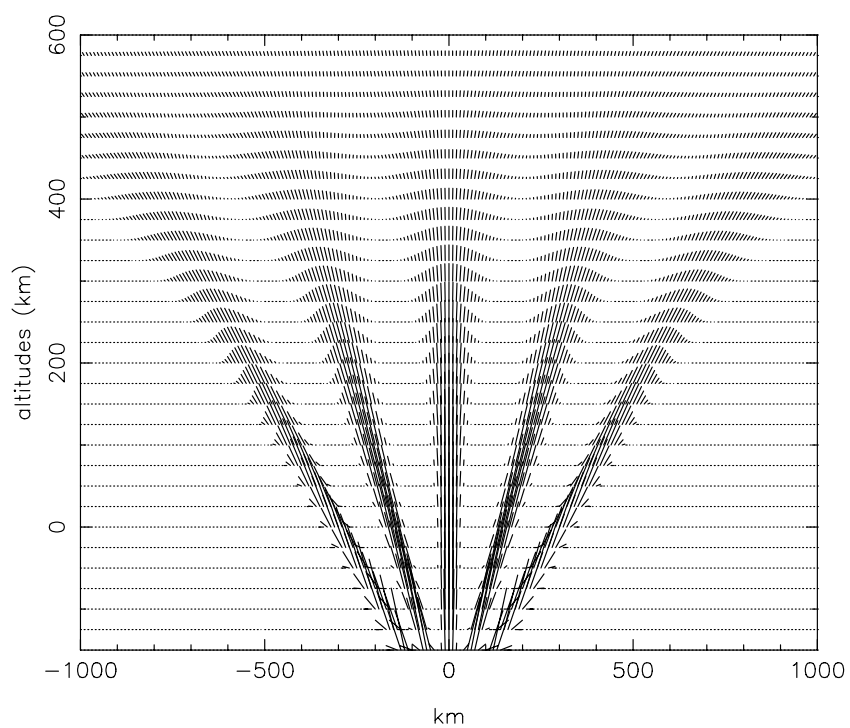

Fig. 16. Magnetic field as in Fig. 7, but for model (III).

where $x_{0}$ is the abscissa of tube axis at altitude $z$. After adding the magnetic fields of all tubes, we define the total pressure by

$P(x, z)=P(-\infty, z)-\left(B_{x}^{t}(x, z)^{2}+B_{z}^{t}(x, z)^{2}\right) / 2 \mu_{0}$

where $B_{x}^{t}$ and $B_{z}^{t}$ are the components of the total field.

Figure 16 shows the magnetic field model (III) with

$R=0.5$

$d(0)=20 \mathrm{~km}$.

Figure 17 shows acceleration vectors. They are larger than in the case of single flux tubes, but do not exceed generally the solar gravity in the slabs where the $\mathrm{NaD}_{1}$ line is formed.

Figure 18 shows the synthetic LOS magnetic field with $s=700 \mathrm{~km}$. As in Sect. 7, synthetic $\mathrm{D}_{1}$ profiles are computed with the MULTI code, by assuming the same VAL3C temperature inside and outside magnetic tubes. Since lines of sight cross several flux tubes, magnetic fields are far from linear functions along each line, and formation altitudes such as altitudes plotted in Fig. 8 are meaningless. The radiative transfer across flux tubes, not parallel to lines of sight, was investigated in the case of iron lines by Audic (1991), who pointed out the strong absorption by quiet regions between flux tubes. This effect is taken into account in our simulation. Because of the width of the $\mathrm{D}_{1}$ line, we again use the weak-field approximation.

The agreement between synthetic and observed values (Fig. 6) is better than with models (I) and (II). Indeed, not only are Eqs. (15) and (16) still satisfied, but the widths $W_{s}$, between 1 and $1.5 \mathrm{arcsec}$, lie in the range of observed values. Clusters of thin flux tubes seem to fit observations better than single wider tubes. However, the magnetic field amplitudes $B_{s}$ are still too small by a factor 4 .

It can also be noted that the departures between magnetic fields in line wings and line core are a little smaller in synthetic values than in observed ones. We have seen in Sect. 8.2 that these departures are due to the combined effects of seeing and slopes of line profile. This suggests that temperatures may play an important role in the relative values of the magnetic field in the wings and in the core. Furthermore, sophisticated models should take temperature gradients versus $x$ into account. In this respect,

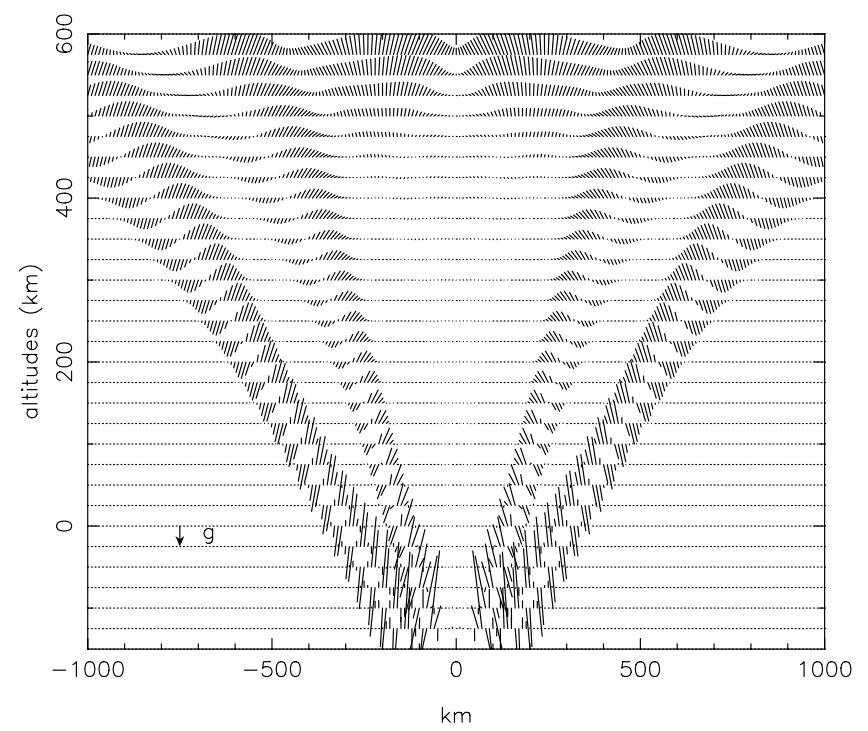

Fig. 17. Acceleration vectors showing the departures from magnetostatic equilibrium as in Fig. 8, but for model (III).

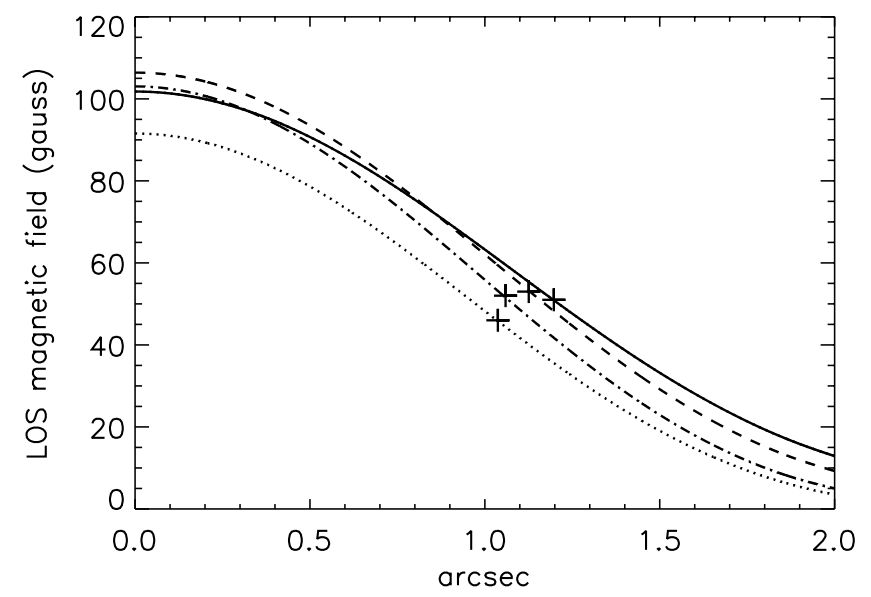

Fig. 18. Synthetic LOS magnetic fields deduced from the flux tube model (III), with seeing effects $(s=700 \mathrm{~km}) . \Delta \lambda$ values are the same as in Fig. 11. Crosses show the points of half maximum values at $W_{s}(\Delta \lambda)$ from central tube axis.

observational constraints might be deduced from intensity observations, such as measurements presented in Fig. 3.

Of course, heat exchanges are connected with velocities of flows along flux tubes. More accurate models should take velocity fields, which can also be deduced from observations, into account (Fig. 4).

Finally, we note that, for a given vertical magnetic field gradient, 3D flux tubes expand more slowly than 2D flux tubes, to keep the flux constant with height, so 3D models would modify the conditions of flux tube merging. They might be investigated for better agreements with observations.

\section{Conclusion}

In this paper, we described observational results and numerical simulations using flux tube models. We used THEMIS/MSDP data to investigate fine structures in the range of altitudes covered by the $\mathrm{NaD}_{1}$ line profile. Intensities, velocities, and LOS magnetic fields were determined across several structures. 
In Sects. 2 and 3, we characterised magnetic and nonmagnetic bright features by simple thermodynamical criteria:

- Velocities are downward in magnetic features, while they are upward in non-magnetic ones.

- Differences between relative intensities in line wings and line core are smaller for magnetic features than for non-magnetic ones.

In the future, such results might be used in two directions. At first, observed velocities can help to build dynamical models with strong departures from equilibrium, while observed intensities can help to check temperatures versus altitude, which control the shape of line profiles. Secondly, we can remember that observations without polarization analysis are possible with shorter exposure-times, so they can lead to higher spatial resolution spectroscopy, and provide us with useful complementary data for investigating temperatures and velocities in fine magnetic flux tubes.

Line-of-sight magnetic fields have been deduced from Zeeman shifts across the $\mathrm{NaD}_{1}$ profile, in simultaneous $(I+V)$ and $(I-V)$ MSDP spectra. As expected, the size of magnetic features are larger in the line core than in the line wings. But other behaviours are more surprising. Magnetic fields measured in the core are larger than in the wings, and the observed magnetic fluxes integrated over the whole features are larger in the core than in the wings.

To understand these results, we simulated observations with two single flux tube models called (I) and (II), using different approaches to the compensation between Lorentz forces and gradients of gas pressure and one conglomerate of thinner flux tubes called model (III). Departures from static equilibrium have been computed. They never exceed the solar gravity in the regions of line formation. Line profile calculations have been computed by the NLTE code MULTI and seeing effects simulated by convolution.

Qualitative agreements with observations have been obtained with all models. For four $\Delta \lambda$-values in the $\mathrm{NaD}_{1}$ profile, both the relative magnetic fields in the centre of magnetic structures and the relative widths of structures, mimic the observations.

From single flux-tube models (I) and (II) by comparing Figs. 6 to 11 and 15, we can draw three conclusions:

- (1) The expansion of flux tubes accounts for the increase in the widths of magnetic features from line wings to line core.

- (2) Departures between slopes of line profiles in the flux tube and the neighbouring quiet sun are different in line core and line wings. On condition that seeing effects result in a small filling factor, they can account for the apparent decrease in magnetic field from line core to line wings close to the axis of the tube.
- (3) The same reasons account for the apparent decrease in the magnetic flux integrated over the whole magnetic features between line core and line wings.

In spite of the qualitative success of models (I) and (II), some problems remain unsolved. The widths of the observed features (Figs. 2 and 6) are larger than the widths of synthetic ones (Figs. 11 and 15), and the amplitudes of observed magnetic fields are larger than synthetic ones.

To try to solve the difficulties, we investigated model (III), which consists of 5 inclined thinner flux-tubes. This model provides synthetic magnetic fields closer to observed ones, at least with respect to the widths of magnetic features.

In this paper, we have confirmed that observed magnetic fields of fine structures cannot be understood without detailed analysis of line formation and filling-factor effects. We have seen also that many observational constraints can be derived, in a wide range of altitudes, from the full profiles of strong lines. MSDP spectroscopy of $\mathrm{NaD}_{1}$, which provides high spatial resolution in spectro-polarimetry, is a powerful tool in this respect.

In addition to new high resolution observations, we think that further investigations of fine magnetic flux tubes should use more sophisticated MHD models, taking thermal and dynamic observational constraints into account.

Acknowledgements. THEMIS is a French-Italian telescope operated on the island of Tenerife by CNRS-CNR in the Spanish Observatorio del Teide of the Instituto de Astrofisica de Canarias. We would like to thank the THEMIS team who operates the telescope at Tenerife, and also C. Coutard and R. Hellier who performed many adjustments of the MSDP. We also thank the referee who proposed many improvements on the first version of this paper.

\section{References}

Audic, S. 1991, Sol. Phys., 135, 275

Beckers, J. M., \& Milkey, R. W. 1975, Sol. Phys., 43, 289

Berlicki, A., Mein, P., \& Schmieder, B. 2006, A\&A, 445, 1127

Carlsson, M., 1986, Uppsala Astron. Obs. Rep. 33

Eibe, M. T., Mein, P., Roudier, Th., Faurobert, M. 2001, A\&A, 371, 1128

Eibe, M. T., Aulanier, A., Faurobert, M., Mein, P., \& Malherbe, J.-M. 2002, A\&A, 381, 290

Leka, K. D., \& Metcalf, T. R. 2003, Sol. Phys., 212, 361

Malherbe, J-M., Roudier, Th., Mein, P., Moity, J., \& Muller, R. 2004, A\&A, 427, 745

Mein, P. 1971, Sol. Phys., 20, 3

Mein, P. 2002, A\&A, 381, 271

Okunev, O. V., \& Kneer, F. 2005, A\&A, 439, 323

Rimmele, T. R. 2004, ApJ, 604, 906

Roudier, Th., Malherbe, J-M., Moity, J., et al. 2006, A\&A, 455, 1091

Shelyag, S., Schüssler, M., Solanki, S. K., Berdyugina, S. V., \& Vögler, A. 2004, A\&A, 427, 335

Solanki, S. K., Finsterle, W., Rüedi, I., \& Livingston, W. 1999, A\&A, 347, L27

Uitenbroek, H. 2003, ASP Conf. Ser. 286, 403

Vernazza, J. E., Avrett, E. H., \& Loeser, R., 1981, ApJS, 45, 635

Vögler, A., Shelyag, S., Schüssler, M., et al. 2005, A\&A, 429, 335 